\title{
MULTIPLICATION OPERATORS ON WEIGHTED SPACES OF VECTOR-VALUED CONTINUOUS FUNCTIONS
}

\author{
R. K. SINGH AND JASBIR SINGH MANHAS
}

(Received 5 June 1989)

Communicated by A. J. Pryde

\begin{abstract}
If $V$ is a system of weights on a completely regular Hausdorff space $X$ and $E$ is a locally convex space, then $C V_{0}(X, E)$ and $C V_{b}(X, E)$ are locally convex spaces of vector-valued continuous functions with topologies generated by seminorms which are weighted analogues of the supremum norm. In this paper we characterise multiplication operators on these spaces induced by scalar-valued and vector-valued mappings. Many examples are presented to illustrate the theory.
\end{abstract}

1980 Mathematics subject classification (Amer. Math. Soc.) (1985 Revision): 47 B 38, 46 E 40.

\section{Introduction}

Let $X$ be a non-empty set, let $E$ be a topological algebra and let $T(X, E)$ be a topological vector space of functions from $X$ to $E$. Let $\theta: X \rightarrow \mathbb{C}$ and $\psi: X \rightarrow E$ be two mappings. Then scalar multiplication and multiplication in $E$ give rise to two linear transformations $M_{\theta}$ and $M_{\psi}$ from $T(X, E)$ to the linear space $L(X, E)$ of all functions from $X$ to $E$, defined as $M_{\theta} f=$ $\theta \cdot f$ and $M_{\psi} f=\psi f$, where the product of functions is defined pointwise. In case $M_{\theta}$ and $M_{\psi}$ take $T(X, E)$ into itself and they are continuous, they are called multiplication operators on $T(X, E)$ induced by $\theta$ and $\psi$ respectively. These operators have been the subject matter of study for a long time on different function spaces, especially on $L^{p}$-spaces, and they have played a very important role in the study of operators on Hilbert spaces.

(C) 1991 Australian Mathematical Society 0263-6115/91 \$A2.00+0.00 
In this note we have endeavoured to study multiplication operators on those weighted spaces of scalar-valued and vector-valued continuous functions which come in contact with topological dynamics.

\section{Preliminaries}

Let $X$ be a completely regular Hausdorff space and $E$ a Hausdorff locally convex topologial vector space over $\mathbb{C}$. Let $c s(E)$ be the set of all continuous seminorms on $E$. By $C(X, E)$ we mean the collection of all continuous functions from $X$ into $E$.

A non-negative upper-semicontinuous function on $X$ will be called a weight on $X$. If $V$ is a set of weights on $X$ such that, given any $x \in X$, there is some $v \in V$ for which $v(x)>0$, we write $V>0$. A set $V$ of weights on $X$ is said to be directed upward provided that, for every pair $v_{1}, v_{2} \in V$ and $\lambda>0$, there exists $v \in V$ so that $\lambda v_{i} \leq v$ (pointwise on $X$ ) for $i=1,2$. We hereafter assume that sets of weights are directed upward. A set $V$ of weights on $X$ which additionally satisfies $V>0$ will be referred to as a system of weights on $X$.

Now, taking a system $V$ of weights on $X$ and a locally convex space $E$, we consider the following vector spaces of continuous functions associated with the triple $(X, V, E)$ :

$C V_{0}(X, E)=\{f \in C(X, E): v f$ vanishes at infinity on $X$ for all $v \in V\}$;

$C V_{b}(X, E)=\{f \in C(X, E): v f(X)$ is bounded in $E$ for all $v \in V\}$.

Obviously $C V_{0}(X, E), C V_{b}(X, E)$ are vector spaces and $C V_{0}(X, E) \subseteq$ $C V_{b}(X, E)$. Now, let $v \in V, q \in c s(E)$ and $f \in C(X, E)$. If we put

$$
P_{v, q}(f)=\operatorname{Sup}\{v(x) q(f(x)): x \in X\}
$$

then $P_{v, q}$ can be regarded as a seminorm on either $C V_{b}(X, E)$ or $C V_{0}(X, E)$, and the family $\left\{P_{v, q}: v \in V, q \in c s(E)\right\}$ defines the locally convex topology on each of these two spaces.

In case $E=\mathbb{C}$, we will omit $E$ from our notation and write $C V_{0}(X)$ in place of $C V_{0}(X, \mathbb{C})$. We also write $P_{v}$ in place of $P_{v, q}$ for each $v \in V$, where $q(z)=|z|, z \in \mathbb{C}$. Moreover, if $E=(E, q)$ is any normed linear space and $v \in V$, we write $P_{v}$ instead of $P_{v, q}$. We shall denote by $B_{v, q}$ the closed unit ball corresponding to the seminorm $P_{v, q}$. In case $E=(E, q)$, we simply write $B_{v}$.

If $U$ and $V$ are two systems of weights on $X$, we write $U \leq V$ whenever given $u \in U$, there exists $v \in V$ such that $u \leq v$. In this case, we then clearly have that $C V_{0}(X, E) \subseteq C U_{0}(X, E)$ and $C V_{b}(X, E) \subseteq C U_{b}(X, E)$, 
as well as that the inclusion map is continuous in both instances. If $U \leq V$ and $V \leq U$, then $U$ and $V$ are equivalent systems of weights on $X$ and we denote this by $U \approx V$.

The spaces $C V_{0}(X)$ and $C V_{b}(X)$ were first introduced by Nachbin [6] and $C V_{0}(X, E), C V_{b}(X, E)$ were subsequently considered in detail by Bierstedt [2] and Prolla [7].

Now, we shall give some examples of these spaces. Let $X$ be a completely regular Hausdorff space. We denote by $\chi_{S}$, the characteristic function of a subset $S$ of $X$. We distinguish four systems of weights on $X$, namely

$U=\left\{\lambda \chi_{K}: \lambda>0, K \subset X, K\right.$ compact $\}$,

$U^{\prime}=C_{c}^{+}(X)$, the set of all positive continuous functions with compact supports,

$V=K^{+}(X)$, the set of all positive constant functions on $X$ and

$V^{\prime}=C_{0}^{+}(X)$, the set of all positive continuous functions vanishing at infinity. Further, if $E$ is a locally convex space, then we define

$$
\begin{aligned}
& C_{0}(X, E)=\{f \in C(X, E): f \text { vanishes at infinity on } X\}, \\
& C_{b}(X, E)=\{f \in C(X, E): f(X) \text { is bounded in } E\} .
\end{aligned}
$$

EXAMPLE. Let $X$ be a completely regular Hausdorff space and let $E$ be a locally convex space. Then

(i) $C U_{0}(X, E)=C U_{b}(X, E)=(C(X, E), k)$ where $k$ denotes the compact open topology,

(ii) $C U_{0}^{\prime}(X, E)=C U_{b}^{\prime}(X, E)=(C(X, E), k)$,

(iii) $C V_{0}(X, E)=\left(C_{0}(X, E), u\right)$ and $C V_{b}(X, E)=\left(C_{b}(X, E), u\right)$, where $u$ denotes the topology of uniform convergence on $X$ and

(iv) $C V_{0}^{\prime}(X, E)=C V_{b}^{\prime}(X, E)=\left(C_{b}(X, E), \beta_{0}\right)$, where $\beta_{0}$ denotes the strict topology.

\section{Functions inducing multiplication operators}

In developing our characterization of those functions $\theta: X \rightarrow \mathbb{C}$ (or $\psi: X \rightarrow E$ ) which induce multiplication operators on weighted spaces of type $C V_{0}(X)$ and $C V_{0}(X, E)$ we work under the following modest requirements.

(2.a) $X$ is a completely regular Hausdorff space.

(2.b) $E$ is a locally convex space such that there exists a vector $s \in E$ for which $p(s) \neq 0$, for every $p \in c s(E)$.

(2.c) $V$ is a system of weights on $X$

(2.d) Corresponding to each $x \in X$, there exists $f_{x} \in C V_{0}(X)$ such that $f_{x}(x) \neq 0$. 
In case $X$ happens to be locally compact, (2.d) is automatically satisfied. For a continuous function $\theta: X \rightarrow \mathbb{C}$ (or $\psi: X \rightarrow E$ ), the set

$$
V|\theta|=\{v|\theta|: v \in V\}
$$

(or for every $p \in \operatorname{cs}(E), V p \circ \psi=\{v p \circ \psi: v \in V\}$ ) is a directed set of weights on $X$ since [9, Theorem 2.2] Summers has shown that the product of two non-negative upper semicontinuous functions is non-negative and upper semicontinuous. In case $\theta: X \rightarrow \mathbb{C}$ (or $\psi: X \rightarrow E$ ) is non-zero at each point of $X, V|\theta|($ or $V p \circ \psi)$ is a system of weights on $X$.

In the following theorem we characterize multiplication operators on $C V_{0}(X)$ induced by scalar-valued functions

2.1 Theorem. Let $\theta: X \rightarrow \mathbb{C}$ be a continuous function. Then $M_{\theta}: C V_{0}(X)$ $\rightarrow C V_{0}(X)$ is a multiplication operator if and only if $V|\theta| \leq V$.

Proof. First, suppose $V|\theta| \leq V$. Then for every $v \in V$, there exists $u \in V$ such that $v|\theta| \leq u$ (pointwise on $X$ ). We show that $M_{\theta}$ is a continuous linear operator on $C V_{0}(X)$. Clearly $M_{\theta}$ is linear on $C V_{0}(X)$. In order to prove the continuity of $M_{\theta}$ on $C V_{0}(X)$, it is enough to show that $M_{\theta}$ is continuous at the origin. For this, suppose $\left\{f_{\alpha}\right\}$ is a net in $C V_{0}(X)$ such that

$$
P_{v}\left(f_{\alpha}\right) \rightarrow 0, \quad \text { for every } v \in V \text {. }
$$

Now,

$$
\begin{aligned}
P_{v}\left(\theta f_{\alpha}\right) & =\operatorname{Sup}\left\{v(x)|\theta(x)|\left|f_{\alpha}(x)\right|: x \in X\right\} \\
& \leq \operatorname{Sup}\left\{u(x)\left|f_{\alpha}(x)\right|: x \in X\right\} \\
& =P_{u}\left(f_{\alpha}\right) \rightarrow 0 .
\end{aligned}
$$

This proves the continuity of $M_{\theta}$ at the origin and hence $M_{\theta}$ is continuous on $C V_{0}(X)$.

Conversely, suppose $M_{\theta}$ is a continuous linear operator on $C V_{0}(X)$. We shall show that $V|\theta| \leq V$. Let $v \in V$. Since $M_{\theta}$ is continuous at the origin, there exists $u \in V$ such that $M_{\theta}\left(B_{u}\right) \subseteq B_{v}$. We claim that $v|\theta| \leq 2 u$. Take $x_{0} \in X$ and set $u\left(x_{0}\right)=\varepsilon$. In case $\varepsilon>0, N=\{x \in X: u(x)<2 \varepsilon\}$ is an open neighbourhood of $x_{0}$. Thus, according to [6, Lemma 2], there exists $f \in C V_{0}(X)$ such that $0 \leq f \leq 1, f\left(x_{0}\right)=1$ and $f(X-N)=0$. Let $g=(2 \varepsilon)^{-1} f$. Then clearly $g \in B_{u}$. Since $M_{\theta}\left(B_{u}\right) \subseteq B_{v}$, we have $\theta g \in B_{v}$ and this yields that

$$
v(x)|\theta(x)||g(x)| \leq 1, \quad \text { for every } x \in X .
$$

From this it follows that

$$
v(x)|\theta(x)||f(x)| \leq 2 \varepsilon, \quad \text { for every } x \in X .
$$


This implies that

$$
v\left(x_{0}\right)\left|\theta\left(x_{0}\right)\right| \leq 2 u\left(x_{0}\right) \text {. }
$$

Now, suppose $u\left(x_{0}\right)=0$ and $v\left(x_{0}\right)\left|\theta\left(x_{0}\right)\right|>0$. If we put $\varepsilon=v\left(x_{0}\right)\left|\theta\left(x_{0}\right)\right| / 2$ and set $N=\{x \in X: u(x)<\varepsilon\}$, then $N$ would be an open neighbourhood of $x_{0}$ and we could again find $f \in C V_{0}(X)$ such that $0 \leq f \leq 1, f\left(x_{0}\right)=1$ and $f(X-N)=0$. Now let $g=\varepsilon^{-1} f$. Then clearly $g \in B_{u}$ and therefore $\theta g \in B_{v}$. Hence

$$
v(x)|\theta(x)||g(x)| \leq 1, \quad \text { for every } x \in X .
$$

This implies that

$$
v(x)|\theta(x)||f(x)| \leq \varepsilon, \quad \text { for every } x \in X .
$$

From this it follows that

$$
v\left(x_{0}\right)\left|\theta\left(x_{0}\right)\right| \leq \frac{v\left(x_{0}\right)\left|\theta\left(x_{0}\right)\right|}{2}
$$

which is impossible. This proves our claim and hence the proof is complete.

Now, we shall characterise multiplication operators on $C V_{0}(X, E)$ induced by scalar-valued functions.

2.2 Theorem. Let $\theta: X \rightarrow \mathbb{C}$ be a continuous function. Then

$$
M_{\theta}: C V_{0}(X, E) \rightarrow C V_{0}(X, E)
$$

is a multiplication operator if and only if $V|\theta| \leq V$.

Proof. First of all, let us suppose $V|\theta| \leq V$. Then for every $v \in V$, there exists $u \in V$ such that $v|\theta| \leq u$ (pointwise on $X$ ). We shall show that $M_{\theta}$ is a continuous linear operator on $C V_{0}(X, E)$. Obviously $M_{\theta}$ is linear on $C V_{0}(X, E)$. It suffices to show that $M_{\theta}$ is a continuous linear operator at the origin. To prove this, let $\left\{f_{\alpha}\right\}$ be a net in $C V_{0}(X, E)$ such that for every $v \in V, q \in \operatorname{cs}(E), P_{v, q}\left(f_{\alpha}\right) \rightarrow 0$. Then

$$
\begin{aligned}
P_{v, q}\left(\theta f_{\alpha}\right) & =\operatorname{Sup}\left\{v(x)|\theta(x)| q\left(f_{\alpha}(x)\right): x \in X\right\} \\
& \leq \operatorname{Sup}\left\{u(x) q\left(f_{\alpha}(x)\right): x \in X\right\} \\
& =P_{u, q}\left(f_{\alpha}\right) \rightarrow 0 .
\end{aligned}
$$

This proves the continuity of $M_{\theta}$ at the origin and hence $M_{\theta}$ is a continuous linear operator on $C V_{0}(X, E)$.

Conversely, suppose $M_{\theta}: C V_{0}(X, E) \rightarrow C V_{0}(X, E)$ is a continuous linear operator. Then we shall show that $V|\theta| \leq V$. Let $v \in V$. Since $M_{\theta}$ is continuous at the origin, therefore for every $v \in V, p \in \operatorname{cs}(E)$, there exists $u \in V, q \in \operatorname{cs}(E)$ such that $M_{\theta}\left(B_{u, q}\right) \subseteq B_{v, p}$. By our assumption 
there exists a vector $s \in E$ such that $p(s) \neq 0$, for every $p \in c s(E)$. Let $\alpha=p(s) / q(s)$. Then $\alpha>0$. We claim that $\alpha v|\theta| \leq 2 u$ (pointwise on $X$ ). Fix $x_{0} \in X$ and set $u\left(x_{0}\right)=\varepsilon$. In case $\varepsilon>0, N=\{x \in X: u(x)<2 \varepsilon\}$ is an open neighbourhood of $x_{0}$ and therefore by [6, Lemma 2] there exists $f \in C V_{0}(X)$ such that $0 \leq f \leq 1, f\left(x_{0}\right)=1$ and $f(X-N)=0$. Define $g(x)=f(x) s$, for every $x \in X$. Then clearly $g \in C V_{0}(X, E)$ and for every $p \in c s(E), 0 \leq(p \circ g) \leq p(s),(p \circ g)\left(x_{0}\right)=p(s)$ and $(p \circ g)(X-N)=0$.

Let $h=(2 \varepsilon)^{-1} g / q(s)$. Then clearly $h \in B_{u, q}$ and this yields that $\theta \cdot h \in$ $B_{v, p}$. Hence $v(x)|\theta(x)| p(h(x)) \leq 1$, for every $x \in X$. From this, it follows that

$$
v(x)|\theta(x)| \frac{1}{q(s)} p(g(x)) \leq 2 \varepsilon, \quad \text { for every } x \in X .
$$

This implies that

$$
v\left(x_{0}\right)\left|\theta\left(x_{0}\right)\right| \frac{p(s)}{q(s)} \leq 2 u\left(x_{0}\right) .
$$

Thus

$$
\alpha v\left(x_{0}\right)\left|\theta\left(x_{0}\right)\right| \leq 2 u\left(x_{0}\right) .
$$

On the other hand, suppose $u\left(x_{0}\right)=0$ and $\alpha v\left(x_{0}\right)\left|\theta\left(x_{0}\right)\right|>0$. Put $\varepsilon=$ $\alpha v\left(x_{0}\right)\left|\theta\left(x_{0}\right)\right| / 2$. Then $N=\{x \in X: u(x)<\varepsilon\}$ is an open neighbourhood of $x_{0}$ and therefore again by [6, Lemma 2] there exists an $f \in C V_{0}(X)$ such that $0 \leq f \leq 1, f\left(x_{0}\right)=1$ and $f(X-N)=0$. Again, define $g(x)=$ $f(x) s$, for every $x \in X$. Then $g \in C V_{0}(X, E)$ and for every $p \in c s(E)$, $0 \leq(p \circ g) \leq p(s),(p \circ g)\left(x_{0}\right)=p(s)$ and $(p \circ g)(X-N)=0$. Consider $h=g / \varepsilon q(s)$. Then $h \in B_{u, q}$ and therefore $\theta h \in B_{v, p}$. Hence

$$
v(x)|\theta(x)| p(h(x)) \leq 1, \quad \text { for every } x \in X .
$$

This implies that

$$
v(x)|\theta(x)| \frac{1}{q(s)} p(g(x)) \leq \varepsilon, \quad \text { for every } x \in X .
$$

From this it follows that

$$
v\left(x_{0}\right)\left|\theta\left(x_{0}\right)\right| \frac{p(s)}{q(s)} \leq \frac{p(s)}{q(s)} \frac{v\left(x_{0}\right)\left|\theta\left(x_{0}\right)\right|}{2} .
$$

Thus $\alpha v\left(x_{0}\right)\left|\theta\left(x_{0}\right)\right| \leq \alpha v\left(x_{0}\right)\left|\theta\left(x_{0}\right)\right| / 2$, which is impossible. Hence our claim is established and the proof is completed.

In order to prove the next theorem, we shall need the following definitions.

Let $E$ be a locally convex algebra with jointly continuous multiplication. It clearly follows that for each $p \in \operatorname{cs}(E)$, there exists a $q \in \operatorname{cs}(E)$ such that $p(x y) \leq q(x) q(y)$, for every $x, y \in E$. A seminorm $p$ on $E$ is said to be submultiplicative if $p(x y) \leq p(x) p(y)$, for every $x, y \in E$. In [5] Michael defines $E$ to be a locally multiplicatively convex algebra, or in short 
an lmc algebra, if there exists a base of neighbourhoods of zero consisting of idempotent absolutely convex sets, or equivalently if its topology is defined by a collection of submultiplicative seminorms. Clearly, multiplication in an lmc algebra is always jointly continuous. For more details and examples of Imc algebras we refer to [3] and [5]. Let $\mathscr{P}$ be a family of submultiplicative seminorms inducing the topology of $E$. Then $\mathscr{P}$ is a subfamily of $\operatorname{cs}(E)$. In [10, c.2.3] Zelazako has noted that for any $\operatorname{lmc}$ algebra $E$ with unit $e$, the family $\mathscr{P}$ can be chosen in such a way that $p(e)=1$, for every $p \in \mathscr{P}$. So we can assume that $\mathscr{P}$ is such a family in this case.

Now, we shall give a characterisation of multiplication operators on $C V_{0}(X, E)$ induced by vector-valued functions.

2.3 TheOREM. Let $E$ be a (locally multiplicatively convex) $\operatorname{lmc}$ algebra with unit $e$ and let $\psi: X \rightarrow E$ be a continuous function. Then

$$
M_{\psi}: C V_{0}(X, E) \rightarrow C V_{0}(X, E)
$$

is a multiplication operator if and only if $V p \circ \psi \leq V$, for every $p \in \mathscr{P}$.

Proof. Suppose $V p \circ \psi \leq V$, for every $p \in \mathscr{P}$. Then for every $v \in V$, there exists $u \in V$ such that $v p \circ \psi \leq u$ (pointwise on $X$ ). We shall show that the mapping $M_{\psi}: C V_{0}(X, E) \rightarrow C(X, E)$, defined by $M_{\psi} f=\psi f$, where product is pointwise, is a continuous linear operator on $C V_{0}(X, E)$. We shall establish the continuity of $M_{\psi}$ at the origin. For this, let $\left\{f_{\alpha}\right\}$ be a net in $C V_{0}(X, E)$ such that for every $v \in V, q \in \mathscr{P}, P_{v, q}\left(f_{\alpha}\right) \rightarrow 0$. Then

$$
\begin{aligned}
P_{v, q}\left(\psi f_{\alpha}\right) & =\operatorname{Sup}\left\{v(x) q\left(\psi(x) f_{\alpha}(x)\right): x \in X\right\} \\
& \leq \operatorname{Sup}\left\{v(x) q(\psi(x)) q\left(f_{\alpha}(x)\right): x \in X\right\} \\
& \leq \operatorname{Sup}\left\{u(x) q\left(f_{\alpha}(x)\right): x \in X\right\} \\
& =P_{u, q}\left(f_{\alpha}\right) \rightarrow 0 .
\end{aligned}
$$

This proves that $M_{\psi}$ is continuous at the origin and hence a continuous linear operator on $C V_{0}(X, E)$.

Conversely, suppose $M_{\psi}: C V_{0}(X, E) \rightarrow C V_{0}(X, E)$ is a continuous linear operator. We shall show that $V p \circ \psi \leq V$, for every $p \in \mathscr{P}$. Let $v \in V$ and $p \in \mathscr{P}$. Since $M_{\psi}$ is continuous at the origin, there exist $u \in V$ and $q \in \mathscr{P}$ such that $M_{\psi}\left(B_{u, q}\right) \subseteq B_{v, p}$. We claim that $v p \circ \psi \leq 2 u$ (pointwise on $X$ ). Fix $x_{0} \in X$ and set $u\left(x_{0}\right)=\varepsilon$. In case $\varepsilon>0$, $N=\{x \in X: u(x)<2 \varepsilon\}$ is an open neighbourhood of $x_{0}$ and therefore according to [6, Lemma 2] there exists $f \in C V_{0}(X)$ such that $0 \leq f \leq 1$, $f\left(x_{0}\right)=1$ and $f(X-N)=0$.

Define $g(x)=f(x) e$, for every $x \in X$, where $e$ is the unit in $E$. Then $g \in C V_{0}(X, E)$ and for every $p \in \mathscr{P}, 0 \leq(p \circ g) \leq 1,(p \circ g)\left(x_{0}\right)=1$ and 
$(p \circ g)(X-N)=0$. If $h=(2 \varepsilon)^{-1} g$, then $h \in B_{u, q}$ and hence $\psi h \in B_{v, p}$. From this it follows that $v(x) p(\psi(x) h(x)) \leq 1$, for every $x \in X$. This implies that

$$
v(x) p(\psi(x) f(x) e) \leq 2 \varepsilon, \quad \text { for every } x \in X .
$$

Thus $v\left(x_{0}\right) p\left(\psi\left(x_{0}\right)\right) \leq 2 u\left(x_{0}\right)$. On the other hand, suppose $u\left(x_{0}\right)=0$ and $v\left(x_{0}\right) p\left(\psi\left(x_{0}\right)\right)>0$. Set $\varepsilon=v\left(x_{0}\right) p\left(\psi\left(x_{0}\right)\right) / 2$. Then $N=\{x \in X: u(x)<\varepsilon\}$ is an open neighbourhood of $x_{0}$ and therefore again by [6, Lemma 2] there exists $f \in C V_{0}(X)$ such that $0 \leq f \leq 1, f\left(x_{0}\right)=1$ and $f(X-N)=0$.

We define $g(x)=f(x) e$, for evey $x \in X$, where $e$ is the unit in $E$. Then $g \in C V_{0}(X, E)$ and for every $p \in \mathscr{P}, 0 \leq(p \circ g) \leq 1,(p \circ g)\left(x_{0}\right)=1$ and $(p \circ g)(X-N)=0$. Choose $h=\varepsilon^{-1} g$. Then clearly $h \in B_{u, q}$ and therefore $\psi h \in B_{v, p}$. This implies that $v(x) p(\psi(x) h(x)) \leq 1$, for every $x \in X$. From this, it follows that $v(x) p(\psi(x) g(x)) \leq \varepsilon$, for every $x \in X$. Further, we get

$$
v\left(x_{0}\right) p\left(\psi\left(x_{0}\right) f\left(x_{0}\right) e\right) \leq \frac{v\left(x_{0}\right) p\left(\psi\left(x_{0}\right)\right)}{2} .
$$

Thus $v\left(x_{0}\right) p\left(\psi\left(x_{0}\right)\right) \leq v\left(x_{0}\right) p\left(\psi\left(x_{0}\right)\right) / 2$ which is impossible and this establishes our claim. This completes the proof of the theorem.

2.4 Remark. Note that if $\theta: X \rightarrow \mathbb{C}$ (or $\psi: X \rightarrow E$ ) is a bounded continuous complex-valued (or vector-valued) function on $X$, then clearly $M_{\theta}$ (or $M_{\psi}$ ) is a multiplication operator on $C V_{0}(X)$ (or $C V_{0}(X, E)$ ) for any system of weights $V$.

If $V$ is the system of weights generated by the characteristic functions of compact sets, then it turns out that every continuous map induces a multiplication operator. This we shall establish in the following theorem.

2.5 THEOREM. Let $X$ be a completely regular Hausdorff space and let

$$
V=\left\{\lambda \chi_{K}: \lambda>0 \text { and } K \subset X, K \text { compact }\right\} \text {. }
$$

(i) Every continuous $\theta: X \rightarrow \mathbb{C}$ induces a multiplication operator on $C V_{0}(X)\left(\right.$ or $\left.C V_{0}(X, E)\right)$.

(ii) Every continuous $\psi: X \rightarrow E$, a locally convex algebra with jointly continuous multiplication, induces a multiplication operator $M_{\psi}$ on $C V_{0}(X, E)$

Proof. (i) In order to prove that $M_{\theta}$ is a continuous linear operator on $C V_{0}(X)$ (or $C V_{0}(X, E)$ ), it is enough to show that for every $v \in V$, there exists $u \in V$ such that $v|\theta| \leq u$ (pointwise on $X$ ). Let $v \in V$. Then $v=\lambda \chi_{K}$, where $K$ is a compact subset of $X$. Let $m=\operatorname{Sup}\{|\theta(x)|: x \in K\}$ and choose $u=\lambda m \chi_{K}$. Then $u \in V$. Since $|\theta(x)| \leq m$, for every $x \in K$, we have

$$
\lambda \chi_{K}(x)|\theta(x)| \leq \lambda m \chi_{K}(x), \quad x \in K
$$


Hence $v(x)|\theta(x)| \leq u(x)$, for every $x \in K$. If $x \in X-K$, then the above inequality is obviously true. Thus we have shown that $v(x)|\theta(x)| \leq u(x)$, for every $x \in X$, and hence by Theorems 2.1 and 2.2, it follows that $M_{\theta}$ is a multiplication operator on $C V_{0}(X)$ (or $C V_{0}(X, E)$ ).

(ii) In view of Theorem 2.3 , it is sufficient to establish the inequality $V p \circ$ $\psi \leq V$, for every $p \in \operatorname{cs}(E)$, that is, for every $v \in V$, there exists $u \in V$ such that $v p \circ \psi \leq u$ (pointwise on $X$ ). Let $v \in V$ and $p \in \operatorname{cs}(E)$. Then $v=\lambda \chi_{K}, K$ a compact subset of $X$. Let $m=\operatorname{Sup}\{p(\psi(x)): x \in K\}$ and choose $u=\lambda m \chi_{K}$. Then $u \in V$. Since $p(\psi(x)) \leq m, x \in K$, we have

$$
\lambda \chi_{K}(x) p(\psi(x)) \leq \lambda m \chi_{K}(x), \quad x \in K .
$$

This implies that $v(x) p(\psi(x)) \leq u(x)$, for every $x \in K$. If $x \in X-K$, then obviously $v(x) p(\psi(x)) \leq u(x)$. Thus $v(x) p(\psi(x)) \leq u(x)$, for every $x \in X$. This completes the proof of the theorem.

2.6 Corollary. Let $X$ have the discrete topology and $V=\left\{\lambda \chi_{K}: \lambda \geq 0\right.$, $K \subset X, K$ a finite set $\}$. Then every function $\theta: X \rightarrow \mathbb{C}($ or $\psi: X \rightarrow E)$ induces a multiplication operator $M_{\theta}\left(\right.$ or $\left.M_{\psi}\right)$ on $C V_{0}(X)\left(\right.$ or $\left.C V_{0}(X, E)\right)$.

2.7 REMARK. (i) In Theorem 2.5, if we replace the system of weights $V=$ $\left\{\lambda \chi_{K}: \lambda>0, K \subset X, K\right.$ compact $\}$ by $U=C_{c}^{+}(X)$, the set of all positive continuous functions on $X$ with compact supports, then the new result is also true.

(ii) If $X$ is a locally compact space, then $V=\left\{\lambda \chi_{K}: \lambda \geq 0, K \subset X\right.$, $K$ compact subset of $X\}$ and $U=C_{c}^{+}(X)$ are equivalent, and otherwise $V \leq U$.

(iii) In Theorems 2.1-2.3 and 2.5 if we replace $C V_{0}(X)$ and $C V_{0}(X, E)$ by $C V_{b}(X)$ and $C V_{b}(X, E)$, then all the new results are also true.

(iv) From Theorem 2.5, we noted that if $\theta: X \rightarrow \mathbb{C}$ (or $\psi: X \rightarrow E$ ) is an unbounded continuous function, even then $\theta$ (or $\psi$ ) gives rise to a multiplication operator $M_{\theta}$ (or $M_{\psi}$ ) on $C V_{0}(X)$ (or $C V_{0}(X, E)$ ). For instance, the polynomial functions on $\mathbb{R}$ induce continuous linear operators on $C V_{0}(\mathbb{R})$, where $V=\left\{\lambda \chi_{K}: \lambda>0, K \subset \mathbb{R}, K\right.$ compact $\}$

Now, we give certain examples of functions which do not induce multiplication operators.

2.8 EXAMPLE. Let $v: \mathbb{N} \rightarrow \mathbb{R}^{+}$be defined as $v(n)=n$, for every $n \in \mathbb{N}$ and let $V=\{\lambda v: \lambda \geq 0\}$. Then $V$ is a system of weights on $\mathbb{N}$ with discrete topology. Let $\theta: \mathbb{N} \rightarrow \mathbb{C}$ be defined as $\theta(n)=n$, for every $n \in \mathbb{N}$. Then $v|\theta|$ is a system of weights on $\mathbb{N}$ and $V|\theta| \Varangle V$. Thus $M_{\theta}$ is not a multiplication operator on $C V_{0}(\mathbb{N})$. In fact, $M_{\theta}$ is not even an into map. To see this, let $f(n)=1 / n^{2}$. Then $f \in C V_{0}(\mathbb{N})$ but $\theta f \notin C V_{0}(\mathbb{N})$. 
2.9 EXAMPLE. Let $\mathbb{N}$ be the set of natural numbers with discrete topology and $V$ be the system of positive constant weights on $\mathbb{N}$. Then $C V_{0}(\mathbb{N})=C_{0}$, the Banach space of all null sequences of complex numbers. Let $\theta: \mathbb{N} \rightarrow \mathbb{C}$ be the identity map. Then $V|\theta| \$ V$ and $M_{\theta}$ is not a multiplication operator on $C_{0}$. Moreover, $M_{\theta}$ is not even an into map. If $f(n)=1 / n$, then $f \in C_{0}$ but $\theta f \notin C_{0}$.

2.10 EXAMPLE. Let $\mathbb{R}^{+}$be the set of positive reals with usual topology and let $v: \mathbb{R}^{+} \rightarrow \mathbb{R}^{+}$be defined as $v(x)=1 / x$, for every $x \in \mathbb{R}^{+}$. Let $V=\{\lambda v: \lambda \geq 0\}$ and let $\theta: \mathbb{R}^{+} \rightarrow \mathbb{C}$ be defined as $\theta(x)=x^{2}$. Then $\theta$ does not induce a multiplication operator $M_{\theta}$ on $C V_{0}\left(\mathbb{R}^{+}\right)$.

\section{References}

[1] K. D. Bierstedt, 'Gewichtete Raume Stetiger Vektorwertiger Funktionen und das Injektive Tensor-produkt I', J. Reine Angew. Math. 259 (1973), 186-210.

[2] K. D. Bierstedt, 'Gewichtete Raume Stetiger Vektorwertiger Funktionen und das Injektive Tensor-produkt II', J. Reine Angew. Math. 260 (1973), 133-146.

[3] A. C. Cochran, R. Keown and C. R. Williams, 'On a class of topological algebras', Pacific J. Math. 34 (1970), 17-25.

[4] G. Kothe, Topological vector spaces I, (Springer-Verlag, Berlin, 1969).

[5] E. A. Michael, 'Locally multiplicatively convex topological algebras', Mem. Amer. Math. Soc. 11 (1952).

[6] L. Nachbin, Elements of approximation theory, (Math. Studies 14, Van Nostrand, Princeton, N.J., 1967).

[7] J. B. Prolla, 'Weighted spaces of vector-valued continuous functions', Ann. Mat. Pura. Appl. (4) 89 (1971), 145-158.

[8] R. K. Singh and W. H. Summers, 'Composition operators on weighted spaces of continuous functions', J. Austral. Math. Soc. (Ser. A) 45 (1988), 303-319.

[9] W. H. Summers, Weighted locally convex spaces of continuous functions, (Ph.D. Dissertation, Louisiana State Univ., 1968).

[10] W. Zelazako, Banach algebras, (Elsevier Publishing Company, PWN, 1973).

\section{University of Jammu}

Jammu 180001

India 